\title{
Apuntes sobre la lectura. El aporte de las ciencias cognitivas
}

\author{
Notes on reading. The contribution of the cognitive sciences
}

\section{GIOVANNA ZAGANELLI}

Università per Stranieri di Perugia

Italia

\begin{abstract}
Resumen. Este trabajo es una contribución a la definición de la lectura a la luz de las teorías semióticas y de las ciencias cognitivas. La operación de lectura es vista como una actividad de decodificación de palabras y signos gráficos individuales. Esto se haría, según los psicólogos cognitivos, a través de una conversión de las células del cerebro programadas para actividades cognitivas diferentes de la lectura. En definitiva, la lectura puede ser definida como una serie de movimientos cognitivos que identifican e interpretan los símbolos graficos incluyéndolos en una secuencia lógica y temporal.
\end{abstract}

Palabras clave: lectura, escritura, cerebro, imagen, narración

\begin{abstract}
This work is a contribution to the definition of reading through the perspective of semiotics and cognitive sciences. In this approach the action and skill of reading is seen both as the activity of decoding the individual words and individual graphic symbols. According to cognitive psychologists and neuroscientists, this happens through a conversion of the brain cells programmed for cognitive tasks other than reading. In essence, reading can be defined through a series of cognitive operations which first identify graphic symbols and then interpret them by placing them in a logical/temporal progression.
\end{abstract}

Keywords: reading, writing, brain, image, narrative

1 Álabe $\mathrm{n}^{\mathrm{o}} 3$ - junio 2011

Para citar este artículo: Zaganelli, G. (2011). Apuntes sobre la lectura. El aporte de las ciencias cognitivas. Álabe, 3. [http://www.ual.es/alabe] 


\section{Introducción}

El objetivo de esta breve aportación es el de contribuir a una definición de lectura, a la luz de algunos recientes estudios producidos en el campo de las ciencias cognitivas y de la semiótica.

En ámbito semiótico, según las teorías de la recepción y de la interpretación, leer una palabra muy simple o un texto literario, significa activar no sólo los significados más inmediatos, sino también formular asociaciones menos frecuentes, o connotaciones.

El texto, en efecto, todo texto, se presenta al lector como un conjunto de signos, los cuales conllevan un significado de carácter lingüístico, denotativo (como ya señaló Barthes y antes de él Hjelmslev), y además un segundo plano de significados culturalmente estratificados. Los signos, sin embargo, se perpetuarían como puros trazos impresos sobre un papel si no fueran activados por el empeño puesto por parte del lector.

La pregunta que surge espontánea en los estudiosos de distintas disciplinas es: ¿Cómo hacemos para llevar a cabo con facilidad y naturaleza operaciones así complejas?

\section{El lector decodificador}

Interesantes respuestas provienen de estudiosos del área cognitiva. Stanislas Dehaene (titular de la cátedra de Psicología cognitiva experimental en el Collège de France), autor de Las neuronas de la lectura, por ejemplo, sostiene que la lectura es el reciclaje, la adaptación de dotaciones presentes en nuestro cerebro de las llamadas neuronas de la lectura. No poseemos, de hecho, estructuras cerebrales apropiadas así como sucede (quizás) para el habla.

¿Pero cuáles son los procesos, según Dehaene, que conducen a la lectura de una palabra? El ojo en un primer momento lee la palabra, luego es confrontada con un archivo lexical, una especie de diccionario que cada uno de nosotros tiene a disposición oculto en el cerebro. A continuación proponemos un ejemplo con las siguientes palabras: tren, estrella, ruda, árbol. 
En el caso en que no se reconociera, o más aún, no se encontrara la palabra - en nuesto caso ruda - entonces se activa la verificación: leemos de nuevo el término, tratamos de interpretarlo a través del contexto y de compararlo con palabras similares.

A un nivel más profundo, según Dehaene, la lectura de la palabra comporta la identificación por parte del ojo y del cerebro de determinadas partes: del prefijo y del sufijo (porción inicial y porción final) y luego del cuerpo central de la palabra. Por ejemplo en la palabra "desabrochar" nos hallaríamos en presencia de la siguiente distribución: des (el prefijo que tiene un significado privativo, quitar), ar (el sufijo que indica que se trata de un verbo) y broche que representa el nombre que constituye el cuerpo central. Se procede, entonces, mediante una operación de separación hasta llegar a la identificación de los elementos mínimos, las letras. Al final todo viene reconstruido en su unidad. No será inútil notar aquí como esta perspectiva pueda tener importantes repercusiones en los estudios de pedagogía de la lectura.

En una línea similar se mueve también Maryanne Wolf, autora de Proust y el calamar (2007) ${ }^{1}$. La estudiosa, neurocientífica cognitiva de la Universidad de Tufts (Massachusetts, EE.UU.), que se ocupa de problemas relacionados con la dislexia, sostiene que la lectura está a cargo de originarias estructuras cerebrales que en el tiempo han experimentado modificaciones y, al hacerlo, han contribuido a cambiar para siempre los circuitos del cerebro y el modo de pensar.

A lo largo de las páginas del libro, la autora demuestra que "no nacimos para leer", o sea, que no existe un área del cerebro predispuesta para la lectura. Por el contrario, distintas áreas de nuestro cerebro son progresivamente utilizadas, y esto porque la especie humana ha aprendido a leer gracias a la plasticidad cerebral, utilizando las capacidades que ya poseía. Por ejemplo se emplean partes del cerebro que son predispuestas al reconocimiento de objetos y a la denominación.

En ambos estudios -el francés y el norteamericano- se muestra una particular atención al nacimiento de la escritura y a la naturaleza de los primeros sistemas gráficos.

A los autores no les es ajeno que el lector mesopotámico (alrededor de 3.500 a.C.) al igual que el egipcio, utilizaba pictogramas sea para referirse a la realidad

\footnotetext{
${ }^{1}$ En castellano responde al título Cómo aprendemos a leer: historia y ciencia del cerebro y la lectura (2008).
} 
circunstante, sea para aludir al concepto que aquel signo significaba, sea con referencia al sonido que aquel signo evocaba. Por lo tanto, en modo complejo, empleaba tanto signos-imagen como signos-sonido (Zaganelli, 2008). Las operaciones de escritura, y de lectura vinculada a ésa, han requerido un notable esfuerzo en este adiestramiento progresivo al signo gráfico, que luego fue compensado con grandes ventajas desde el punto de vista de la evolución con la instauración de nuevas conexiones entre los circuitos neuronales. De particular interés resulta la diferencia que Wolf evidencia entre un lector que utiliza un alfabeto como el inglés, por ejemplo, basado en la abstracción (y por lo tanto en la economicidad lingüística) y un lector de signos ideográficos como el chino y el japonés los cuales activan, a pesar de su complejidad, un empleo más amplio de las áreas visuales.

Las reflexiones que los neurocientíficos han elaborado presentan estrechas vinculaciones con las de los lingüistas y antropólogos que se han ocupado de las relaciones entre oralidad y escritura como, por citar algunos, Ong (1982), Havelock (1963), Goody (1986). La escritura, como ellos han demostrado, ha modificado las modalidades de las actividades intelectuales del hombre: reestructurando las jerarquías sensoriales con la transición de sonido (canales vinculados a ambas culturas primarias y secundarias de la oralidad) para la representación visual (que dan prioridad a la vista sobre el oído) estimulando el razonamiento abstracto y, con ello, la posibilidad de confrontamientos dialécticos, poniendo las bases del campo jurídico y de las leyes. Como explica Goody, “en Europa la distinción entre ley y costumbre se basa en definitiva sobre lo que es escrito y lo que no lo es. Codificar una costumbre significa en primer lugar ponerla por escrito, y después darle fuerza de ley" (Goody 1986, 1988:151).

En el fondo los discursos propuestos por los estudiosos de lengua y de cultura se encuentran hoy en día (sobre todo a partir de los últimos 15-20 años) con los de los estudiosos del cerebro, dando lugar a un destacado número de investigaciones de fundamental importancia para la comprensión de los procesos de lectura, de gran eficacia incluso en el plano pedagógico y para los estudios sobre la dislexia.

Podemos, finalmente, extraer una primera provisoria conclusión sobre la lectura: leer, en cualquier idioma, acarrea transformaciones en el modo de pensar, cambia el cerebro, incrementa las conexiones entre los circuitos cerebrales. Volviendo sobre los 
pasos y teniendo en cuenta la perspectiva cognitiva y la semiótica, podemos identificar las actividades de lectura del modo detallado a continuación:

1. Conectar los signos escritos a sonidos (el más inteligente de los descubrimientos según Wolf) ${ }^{2}$.

2. Reconocer cada uno de los signos gráficos, grupos consonánticos, prefijos, sufijos.

3. Combinar elementos lingüísticos y figurativos. El lector de ayer, mesopotámico y egipcio, al igual que el lector oriental y el occidental de hoy, comparten en la escritura y en la lectura, si bien en medida diferente, experiencias figurativas y lingüísticas.

\section{Del lector descodificador al lector-intérprete: la narración como construcción de sentido}

Una vez que el cerebro es capaz de reconocer configuraciones con una cierta fluidez y desenvoltura, el lector tendrá el tiempo necesario para "ir más allá del texto", podrá dedicarse a proyectar estrategias, formular inferencias, será capaz, en definitiva, de crear nexos entre lo que el texto dice y lo que el texto no dice, entre lo que se manifiesta en superficie y lo que está depositado en su estructura profunda.

Dos son las investigaciones que quisiéramos sugerir: D. Herman $(2002,2003)$ y L. Zunshine (2006). Este ámbito científico estudia las interrelaciones entre las narraciones y la inteligencia, y trata de establecer un nexo entre la teoría de la narración y las ciencias cognitivas. ${ }^{3}$

Herman, profesor de la Ohio State University, sostiene que la narratividad, entendida como competencia narrativa (vivimos inmersos cotidianamente en un universo narrativo - literario, fílmico, cotidiano - que crea horizontes de expectativa),

\footnotetext{
${ }^{2}$ Sobre este punto el debate teórico se abre a posiciones divergentes no del todo conformes a las de los cognitivistas y neurocientíficos: la escritura, según perspectivas lingüísticas (Cardona 1981 y Harris 2000), no importa la forma en la que se presente. Desde los primeros sistemas gráficos hasta el alfabeto griego, representa un sistema a todos los efectos, útil para la comunidad que lo ha producido. Sobre las diferentes posiciones respecto a las escrituras, véase Zaganelli (2008).

${ }^{3}$ Las investigaciones mencionadas fueron precedidas por una infinidad de estudios que centran su atención en la importancia del pensamiento narrativo como instrumento cognitivo que permite identificar e interpretar la realidad. No podemos, a este propósito, dejar de citar como precursores de las antedichas teorías a Vygotsky (1962), Minsky (1963) y Bruner (1991).
} 
es capaz de ofrecer modelos de comprensión conceptual de las situaciones y de cooperar en la construcción espacio-temporal del actuar humano.

¿Qué cosa es y cómo está organizada la narratividad?

Partiendo de reflexiones, hoy en día consolidadas, elaboradas por las ciencias lingüístico-semióticas, por narratividad entendemos la capacidad, casi una exigencia, que tenemos desde la infancia de incluir en una secuencia lógico-temporal los eventos, y de construir una linealidad en la cual causas y efectos están relacionados entre sí. Se trata, en definitiva, de poner orden en el desorden de los eventos. También el nacimiento de la novela corresponde a esta función, así como los géneros literarios. La narración literaria, la novela, representan un componente de la narratividad que se extiende incluso a otros ámbitos como el periódico, el derecho, la publicidad, la política, los medios o el marketing, áreas no consideradas, en general, como típicamente narrativas.

En el campo de la publicidad, la marca, por ejemplo, es siempre menos una puesta en escena del producto y de sus características, para ser más bien una construcción narrativa que engloba en su interior al consumidor y lo introduce en un programa de transformación que lo conduce a la superación de algunas arduas pruebas para llegar al final a alcanzar su objeto de valor, el producto en cuestión. En definitiva leer un texto, aunque no sea exclusivamente del género narrativo, e interpretarlo, significa identificar la estructura narrativa profunda.

Greimas, distinguido representante de la Escuela de París (centro que en los años '60-'70 dio inicio y luego representó un punto de referencia científico fuerte en el debate semiótico) ha analizado no sólo relatos literarios - en particular Maupassant utilizando instrumentos narrativos, sino que además se ha ocupado de discursos jurídicos y hasta de recetas de cocina. Su "famosa" Zuppa al pesto (1979) ${ }^{4}$ es una historia de sujetos que adquieren competencia en la construcción de un objeto de valor, el plato, alcanzando de este modo la performance. Según la hipótesis semiótica, hablar de objeto de valor significa no tanto considerar el objeto en sí, sino más bien como un lugar de investidura de valores, capaz de mediar la relación del sujeto consigo mismo.

\footnotetext{
${ }^{4}$ En castellano: Greimas, A. Julián (1989). La sopa al 'pistou' o la construcción de un objeto de valor. En Del Sentido, II. Madrid: Gredos.
} 
A la luz de estas reflexiones la receta de cocina no debe ser considerada como un texto prescriptivo, como pareciera mostrar su superficie (la presencia constante del imperativo sugeriría este estatuto: "Verted en una olla provenzal 6 litros de agua...., abrid un kilo de judías frescas..., pelad seis patatas...”) (Greimas 1983, 1984: 152), ésa más bien representa un contrato del tipo: «Si se siguen correctamente todas las indicaciones dadas, entonces se obtendrá la sopa al 'pistou'»(Greimas 1983, 984:153). El análisis textual-narrativo tiende a evidenciar la disconformidad entre la organización de la superficie y sus estructuras profundas.

A cumplir, colocándolos en una progresión causal-temporal-modal, los steps que componen el texto: un intinerario sabiamente programado de acciones a cumplir. ${ }^{5}$

\section{Encuentro entre semiótica y ciencias cognitivas}

Los estudios sobre narración, pertenecientes al ámbito semiótico, han encontrado un válido sostén en algunas teorías elaboradas por psicólogos y teóricos del cognitivismo (Bruner, 1993; Herman, 2002, 2003; Zunshine, 2006, entre otros) ${ }^{6}$. Herman (2003) sostiene que el individuo recurre a la narración en diversos contextos siendo el relato un instrumento cognitivo capaz de organizar pensamiento y comportamiento en una gran variedad de actividades humanas. Antes que él Bruner (1991: 4) afirmaba que "Cada uno de nosotros organiza la propia experiencia y la memoria de los sucesos humanos principalmente en forma narrativa - historias, excusas, mitos, razones para hacer o no hacer, etc.".

En la base de la narratividad, según Herman, actúan dos categorías cognitivas: schema (esquema) y script (guión). Con schemata (esquemas) nos referimos a las experiencias que con el paso del tiempo se han ido sedimentando, generando modelos estereotípicos, que pueden naturalmente ser aumentados y modificados a medida que se

\footnotetext{
${ }^{5}$ Generalmente se atribuye a Vladimir Propp, autor de Morfologia della fiaba (1928) [en castellano Morfología del cuento] el origen de las reflexiones sobre el análisis del relato. En la citada obra Propp resalta la existencia de funciones constantes e invariables que se manifiestan en superficie a través de valores variables. A sus estudios se remitieron no sólo Greimas, ya citado, sino también otros narratólogos, como por ejemplo Genette, Bremond, Barthes, Todorov (1966).

${ }^{6}$ Las siguientes observaciones han sido organizadas teniendo en cuenta sobre todo Neuronarratologia. Il futuro dell'analisi del racconto editado por Stefano Calabrese (2009, Bologna, Archetipolibri); Routledge encyclopedia of narrative theory, (ver la voz narración) editado por David Herman, Manfred Jahn. y Marie-Laure Ryan (2005, London-New York: Routledge) y, finalmente La Fabbrica delle storie. Diritto, letteratura, vita de Jerome Bruner (2002, Roma-Bari, Laterza). [en castellano La fábrica de historia : derecho, literatura, vida]
} 
experimentan nuevas vivencias, en base a los cuales leer las nuevas experiencias y orientar nuestro comportamiento. Se trata por lo tanto de informaciones memorizadas en un archivo cognitivo, identificadas (e identificables) semánticamente, como por ejemplo "compras en el supermercado", "lección universitaria", "ceremonia religiosa", etc. Con ellos actúan los "guiones" (scripts) (micro escenificaciones). Estos representan, en cambio, una especie de codificaciones capaces de articular sintácticamente lo que sucede al interno de las clasificaciones abstractas de los datos de la experiencia, los schemata a los que nos hemos referido. Por consiguiente, a modo de ejemplo, el esquema "hacer las compras en el supermercado", comporta los siguientes scripts: aparcar el coche, coger el carrito, introducir la moneda, observar la mercadería en las estanterías, elegir los productos, etc. (Calabrese, 2010).

Los scripts se refieren a procesos dinámicos, son particulares sintagmas que se articulan al interno del paradigma esquemático. Todo, en definitiva, desde las historias de las cuales somos cotidianamente actores, a las informaciones periodísticas o las series televisivas, hasta llegar a los textos literarios, funciona dentro de estos dos ejes representados por los schemata y los scripts. Memoria semántica el primero, memoria episódica o secuencial el segundo.

Al lado de las ciencias cognitivas, las propuestas semiótico-lingüísticas, y las hipótesis antropológicas podemos colocar tambièn las teorías literarias al fin de evaluar la importancia del relato como instrumento del pensamiento. Para concluir cito los estudios efectuados por Lisa Zunshine, profesora de literatura de la Universidad de Kentucky y autora de Why we Read Fiction? Theory of Mind and the Novel (2006).

Es evidente que el relato literario, hecho de actores, eventos, ambientes, enriquece los archivos, las bases de datos a los que nos hemos referido. Este aporta modelos de comprensión conceptual de las situaciones, construye horizontes de expectativas y conduce por el camino que lleva a tomar decisiones para la acción (problem solving). Por otra parte nos estimula a adivinar la finalidad de los personajes presentes en él, a intuir sus pensamientos y además nos ofrece la satisfacción de elaborar soluciones diferentes de las de la vida real. Por esto, las novelas son una herramienta para el desarrollo de nuestra habilidad metarepresentacional: esas, de hecho, son capaces de capturar nuestra atención mostrándonos personas diferentes a las que normalmente tratamos y al mismo tiempo nos desafía a comprenderlas. Por último, 
nos gratifica revelándonos verdaderamente sus pensamientos y sus proyectos. La habilidad metarepresentacional, junto a la Teoría de la mente, son los dos puntos fundamentales de las ciencias cognitivas a través de los cuales Zunshine ha afrontado el análisis de los textos literarios.

Por Teoría de la mente (mind-reading) se entiende la capacidad de los seres humanos de atribuir pensamientos, sentimientos e intenciones a los otros seres humanos siguiendo procedimientos interferenciales. Se trata de habilidades desarrolladas por los individuos en el curso de la evolución con el fin de relacionarse con los otros y de establecer relaciones eficaces en la comunidad social. Por metarepresentationality nos referimos a "informaciones conservadas en base a un indicio" y corregibles o modificables de acuerdo a sucesivas informaciones. Existen entonces diferentes grados de indicios gracias a los cuales conservamos informaciones, construimos las hipótesis e interpretamos los textos.

La lectura, si es observada a través de la lente de los estudios cognitivos, puede ciertamente ser considerada la actividad interpretativa que se aplica al texto literario, pero no puede coincidir exclusivamente con esta operación. Puede ser reconsiderada como un conjunto de procesos, o mejor dicho, una estratificación de procesos, a saber:

1. La identificación por parte del ojo, del cerebro y de la mente de determinados caracteres gráficos (sea en sentido histórico-social, sea en sentido individual).

2. La identificación de la arquitectura narrativa presente en los textos a los que nos sometemos en la vida de cada día y, por lo tanto, la organización espacio-temporal de los eventos.

3. Construcción de hipótesis interpretativas a través de inferencias, activación de connotaciones que permiten la representación imaginativa de situaciones y escenas (sobre todo cuando se leen historias con personajes y móviles).

4. Incremento e integración de los conocimientos que se adquieren con los que ya se poseen.

5. Activación del sistema pasional que permite probar emociones en relación a lo que se lee. 
Se trata de operaciones que no se excluyen recíprocamente, sino más bien que se compenetran en la práctica de la lectura. Esas tienen una importante repercusión también sobre el plano de los textos electrónicos, por ejemplo, donde el uso de caracteres individuales, del lettering, del color, reconquista un espacio de primera importancia, donde la combinación entre lenguaje e imagen representa a menudo la base del texto y donde las narraciones organizan precisos caminos de lectura.

Hoy en día el lector electrónico asume en sí mismo tanto el lector decodificador como el interpretante: al primero le debe la importancia del signo gráfico, al segundo sutiles caminos narrativos ocultos en la combinación de lenguaje e imagen.

\section{Referencias bibliográficas}

Barthes, R., Bremond, C., Genette, G., Todorov, T. et alii (1966). Recherches sémiologiques: l'analyse structurale du récit. Communications, 8.

Bruner, J. (1986). Actual Minds and Possible Worlds. Cambridge Mass.-London: Harvard University Press.

Bruner, J.S. (1991). The narrative construction of reality. Critical Inquiry, 18, 1-21.

Bruner, J.S. (2002). La fabbrica delle storie. Diritto, letteratura, vita. Roma-Bari: Laterza.

Calabrese, S. (2009). Neuronarratologia. Il futuro dell'analisi del racconto. Bologna: Archetipolibri.

Calabrese, S. (2010). La comunicazione narrativa. Dalla letteratura alla quotidianità. Milano: Bruno Mondadori.

Cardona, G.R.(1981). Antropologia della scrittura. Torino: Loescher.

Dehaene, S. (2007). Les neurones de la lecture. Paris: Odile, Jacob.

Goody, J. (1986). The Logic of Writing and the Organization of Society. Cambridge: Cambridge University Press.

Greimas, A.J. (1979). La soupe au pistou ou la construction d'un objet de valeur. Actes sémiotiques - Documents, 5.

Greimas, A.J. (1983). Du sens II - Éssais sémiotiques. Paris : Seuil. (trad. it. Greimas A.J. (1984). Del senso 2. Narrativa, modalità, passioni. Milano: Bompiani)

Harris, R. (2000). Rethinking Writing. London: The Athlone Press.

Havelock, E.A. (1963). Preface to Plato. Cambridge, Mass.: Harvard University Press

Herman, D. (2002). Story Logic Problems and Possibilities of Narrative. Lincoln: University of Nebraska Press.

Herman, D. (Ed.) (2003). Narrative Theory and the Cognitive Sciences . California USA: CSLI Publications. 
Herman, D.; Jahn, M. y Ryan, M-L. (Ed.) (2005). Routledge encyclopedia of narrative theory. London-New York: Routledge.

Minsky, M. (1968). Matter Mind and Models. En M. Minsky. Semantic Information Processing. Cambridge, Mass.: MIT Press.

Ong, W. (1982). Orality and Literacy. The Technologizing of the Word. London-New York: Methuen.

Propp, V. (1928). Morfologia della fiaba. Torino: Giulio Einaudi 1966.

Vygotsky, L. S. (1962). Thought and language. Cambridge, Mass.: MIT Press

Wolf, M. (2007). Proust and the Squid. The Story and Science of the Reading Brain. London: Harper.

Zaganelli, G. (2008). Itinerari dell'immagine. Per una semiotica della scrittura. Milano: Lupetti Editori di Comunicazione.

Zunshine, L. (2006). Why We Read Fiction? Theory of Mind and the Novel. Columbus: Ohio State University Press.

(Artículo enviado: 06-04-2011; revisado: 18-05-2011; aceptado: 18-05-2011) 